\title{
The conformational impact of eliminating the C- terminal peptide extension from IMPDH1- 604 retinal isoform via experimental and molecular dynamics simulation approaches
}

\section{Parisa Elyasi Ebli}

Institute of Biochemistry and Biophysics, the University of Tehran

Razieh Yazdanparast ( $\sim$ ryazdan@ut.ac.ir)

Institute of Biochemistry and Biophysics, the University of Tehran

Sajjad Gharaghani

Institute of Biochemistry and Biophysics, the University of Tehran

\section{Research Article}

Keywords: Retinitis Pigmentosa, Inosine Monophosphate Dehydrogenase, Molecular Dynamics Simulation, Proteolytic Digestion, Retinal Isoform

Posted Date: April 19th, 2022

DOI: https://doi.org/10.21203/rs.3.rs-1246219/v2

License: (c) (1) This work is licensed under a Creative Commons Attribution 4.0 International License.

Read Full License 


\section{Abstract}

Retinitis Pigmentosa, a blindness with acquired and hereditary origins, includes 2 percent of global cases of blindness. RP10, a gene involved in autosomal dominant form of the RP, encodes for isoform 1 of inosine 5'-monophosphate dehydrogenase (IMPDH1). IMPDH1, the rate-limiting enzyme in the purine nucleotide de novo synthesis pathway, retinal isoforms are identified as the root cause of observing diverse phenotypes of IMPDH1 mutations in other tissue cells in comparison to the retinal photoreceptors. Considering previously reported significant varied kinetic activity among retinal isoforms, the central focus of the current article is prediction of possible structural explanations. The recombinant 604-isoform lacking carboxyl terminus peptide fragment has been used in proteolytic digestion with aChymotrypsin as a means of detecting intermolecular interactions. Dimer models of wild type 604isoform were subjected to molecular dynamics (MD) simulation. Our results indicated that a 604-isoform lacking C-terminal peptide tends to have a more accessible surface and thus a more rapid proteolysis. Our computational data predict a possible spontaneous inhibition due to formation of a novel helix in GTP binding site in the engineered protein.

\section{Introduction}

Retinitis pigmentosa (RP), first diagnosed by Donders in 1858, is an acquired and inherited blindness which till date, 13, 21 and 5 genes have been reported to cause the autosomal dominant, autosomal recessive and x-linked inheritance patterns, respectively. Mutations in RP10 gene, coding IMPDH1 (EC: 1.1.1.205), has been accounted for the autosomal dominant form of RP [1]. IMPDH1, the rate-limiting enzyme in purine nucleotide de novo synthesis pathway, catalyzes the conversion of IMP to xanthosine monophosphate (XMP) using a NAD molecule as cofactor [2, 3]. In Mus musculus, in addition to the canonical isoform containing 514 amino acids, retinal isoforms containing 546 and 604 aa have also been reported in mammalians. The 546 isoform has a 32 aa peptide on the carboxyl terminal of the protein sequence. Also, 604-isoform contains an extra 57 aa peptide on the amine terminal in addition to the carboxyl peptide present in both 546- and 604-isoforms. It has been reported that 604-isoform contains 595 aa in Homo sapiens [4, 5].

Structure of IMPDH1 consists of a catalytic domain with $(\beta / a)_{8}$ or TIM barrel conformation and two tandem CBS subdomains. The active site of the enzyme consists of Cys319, located at the carboxyl end of the $\beta$-sheets in a GIGPGSICTT motif [6-8]. catalytic flap, a twisted $\beta$-sheet on the C-terminal of catalytic domain, is of assistance in formation of a pseudo barrel in octameric conformation of the enzyme. This domain is located between $a 8$ and $\beta 8$ of the catalytic domain and has two conserved amino acids, Arg418 and Tyr419, which have been reported to play a crucial role in activating a water molecule essential for catalytic activity of the enzyme [9, 10]. CBS subdomains, responsible for regulation of the activity and formation of octameric structures of the enzyme, possess three nucleotide binding sites. The combination of GDP/GTP or ATP molecules binding to these binding sites have been proven to play a key role in attenuation or elevation of the catalytic activity of the enzyme [11-13]. Also, it has been noted that occupation of first and second binding sites by GDP/GTP molecules results in a decrease in 
catalytic activity [11, 12]. Moreover, among the identified RP-related mutations in IMPDH1, R224P and D226N (HsIMPDH numbering; equivalent to R226P and D228N in Mus musculus) are in nucleotide binding sites of CBS subdomain [11].

On a previous computational study on structural properties of retinal isoforms, the possible role of $\mathrm{C} / \mathrm{N}$ terminal peptides in regulation of catalytic activity has been denoted [14]. In a recent study, it has been shown that a Homo sapiens 595 aa isoform lacking the carboxyl terminal peptide has three-fold decrease in GTP sensitivity due to the formation of the filaments. This study pointed out the filamentation characteristics as the main factor of inhibition and catalytic activity variance in the wild type and engineered protein [15].

A study by Nimmesgern, et. al in 1996 performed proteolysis of IMPDH2 in free and substrate-bound states and concluded a change of proteolytic pattern. Their results demonstrated that an unbound state of IMPDH2 is more susceptible to protease activity than the substrate bound states [16].

Lacking X-ray crystallographic studies on the structure of retinal isoforms, here, in order to investigate the unidentified internal interactions in presence and absence of C-terminal peptide of murine retinal 604isoform, computational approaches were taken. Also, by comparing proteolytic digestion patterns of the recombinant and wild type 604-isoform, possible conformation changes due to the C-terminal peptide were studied.

\section{Methods}

\section{Chemicals and reagents}

All restriction endonucleases, DNA Ligase, Pfu polymerase and a-Chymotrypsin were purchased from Thermo scientific. DNA Ladder and protein size marker were obtained from Thermo scientific. All other chemicals used were from Merck or Sigma.

\section{Cloning and expression of 604-isoform lacking C-terminal peptide}

Using the previously cloned sequence of 604-isoform, the desired protein sequence was amplified with Pfu polymerase, forward primer including Ndel restriction site; $5^{\prime}$ -

GGAATTCCATATGGAGGAACCGCTCTCACC-3' and reverse primer including Xhol restriction site; 5'CCGCTCGAGAAACGGCAGAAAGGTATAGG-3'. The amplified PCR products were cloned to pET26b+ vector and the accuracy of sequence has been assessed and confirmed by DNA sequencing using T7 promoter and T7 terminator primers.

Cloned constructs were transformed into E. coli strain BL21 (DE3) and inoculated into $100 \mathrm{ml}$ of Louria Bertani (LB) medium containing $30 \mu \mathrm{g} / \mathrm{ml}$ kanamycin. Induction was initiated by addition of $1 \mathrm{mM}$ IPTG. 
The mixture kept at $20^{\circ} \mathrm{C}$ for 5 hours while shaking at $200 \mathrm{rpm}$. Harvested cells were stored at $-70{ }^{\circ} \mathrm{C}$.

\section{Purification of recombinant proteins}

Frozen cell pellets were thawed and resuspended in 3-4 $\mathrm{ml}$ of lysis buffer $\left(50 \mathrm{mM} \mathrm{NaH}_{2} \mathrm{PO}_{4}, 300 \mathrm{mM}\right.$ $\mathrm{NaCl}, 10 \mathrm{mM}$ Imidazole). To the bacterial suspension, lysozyme with the final concentration of $30 \mathrm{mg} / \mathrm{ml}$ was added and incubated on ice for 30 minutes. The suspension was sonicated and centrifuged for 30 minutes at $17000 \mathrm{~g}$ and $4{ }^{\circ} \mathrm{C}$. The resultant supernatant was applied to Ni-NTA column and recombinant protein purified following the manufacturer's protocol. The protein quantity was analyzed using Lowry's method [17] with bovine serum albumin as the standard.

\section{Proteolytic digestion}

Proteolysis of the protein samples were performed using a 1:100 molecular weight ratio of aChymotrypsin and the protein. Here, to each reaction containing $50 \mu \mathrm{g}$ of the protein in the elution buffer ( $500 \mathrm{mM}$ Imidazole, $300 \mathrm{mM} \mathrm{NaCl}$ and $50 \mathrm{mM} \mathrm{Na}_{2} \mathrm{HPO}_{4}$ ) and $0.5 \mu \mathrm{g}$ of a-Chymotrypsin in $200 \mu$ lotal volume, for $0,2-, 4-, 6-, 8-, 10$ - and 15-minutes time intervals, $2 \mu$ of $10 \%$ SDS was added to stop the proteolysis. Then, the protein content of each reaction was precipitated by adding $10 \mu \mathrm{l}$ of $3 \mathrm{M} \mathrm{KCl}$ followed by centrifugation for 5 minutes at $15000 \mathrm{~g}$. The resulting precipitate was dissolved in $1 \mathrm{x}$ SDSPAGE loading buffer and subsequently analyzed by a $10 \%$ SDS-PAGE gel.

\section{MD simulation}

Models of engineered protein were built by eliminating the 32 amino acids of C-terminal extension peptide of 604-isoform structure obtained from a previous study modeling monomer and octamers of each retinal isoform [14]. In order to discover the interactions of a convex octamer, a dimer structure of upper and lower monomers of convex octamer were chosen. Using GROMACS 2019.1 simulation package [18] and CHARMM36 force field [19], these models were subjected to molecular dynamics simulation. First, the structures were solvated with TIP3P water model [20] in a cubic box, a $1 \mathrm{~nm}$ distance between protein and box edges. The system was neutralized by adding sufficient counter charge ions. Energy minimization of the system was performed by steepest decent method [21]. This was followed by 200 ps of MD simulation in the canonical (NVT) ensemble using Berendsen-thermostat algorithm [22] to increase the temperature of our system to $300 \mathrm{~K}$, and an additional $200 \mathrm{ps}$ of equilibration under NPT conditions with Parrinello-Rahman algorithm [23]. The production run was implemented for a $20 \mathrm{~ns}$ simulation time, which is sufficient to relax the residue positions. RMSD and RMSF analyses were performed using GROMACS tools. The interactions of $\mathrm{N}$ - and C-terminal peptides were explored visually by UCSF Chimera [24].

\section{Results}




\section{Recombinant production of $\Delta \mathrm{C}$-fragment 604-isoform}

Recombinant 604-isoform lacking C-terminal 32 amino acid peptide was overexpressed in E. coli strain BL21 (DE3) in a pET26b+ vector. A polyHistidine-tag was located at the C-terminal of the desired protein. PCR-sequencing of recombinant vector with T7 promoter and T7 terminator primers confirmed the accuracy of target gene. Optimum expression of both wild type and the modified recombinant proteins were reached at $1 \mathrm{mM} \mathrm{IPTG}$, and shaking for 5 hours at $20^{\circ} \mathrm{C}$.

\section{protease digestion}

In order to study the fluctuations of the 604-isoform structure, proteolytic digestion was carried out. The expressed purified proteins were digested with a-chymotrypsin for $0,2,4,6,8,10$ and 15 minutes and SDS-PAGE analyses were used to assess the resultant bands of the proteolytic digestion. As it is shown in figure 1, the protein bands lower than $35 \mathrm{kDa}$ are the products of digestion, while molecular weight of WT protein is $64 \mathrm{kDa}$. As is shown on figure 1, it is well accepted that the mobility pattern of the digested two samples is different. As in the engineered protein digestion the bands occurred faster and within the first 2 minutes, much more digested fragments $(\mathrm{MW}<35 \mathrm{kDa})$ are formed relative to that of the wild type.

This could be interpreted as increased flexibility and extension of structure due to the C-terminal fragment elimination and more accessibility for a-Chymotrypsin activity.

\section{Simulation}

MD simulation was utilized in order to obtain a mere understanding of the internal interactions of the $C$ and $\mathrm{N}$ terminal peptides with the other domains, both in engineered and wild type 604-isoforms. Here, a dimer of proteins was extracted from a convex octamer structure obtained from a previous study in our lab [15]. The structure of the engineered protein was constructed by eliminating 32 amino acids from the wild type 604-isoform. To evaluate the stability and flexibility, Root Mean Squared Deviation (RMSD) and Root Mean Squared Fluctuation (RMSF) were calculated.

The RMSD of all structures converged after $10 \mathrm{~ns}$ and stayed stable for the remaining time of MD simulation. This suggests that the systems, dimers of both wild type and the engineered protein, reached equilibrium state after $10 \mathrm{~ns}$ (Fig. 2).

Additionally, to investigate mean residual movement over the course of time, the RMSF was calculated for each residue (Fig. 3). As expected, the amino and carboxyl terminal residues of both protein dimers show higher values than the other protein sites. Although lacking the extended C-terminal peptide, the carboxy terminal (C-ter) of the engineered protein (Fig. 2, shown in red and yellow) shows higher degree of mobility compared to the carboxy terminal of the wild type protein (Fig. 2, shown in light and dark green). The higher RMSF value of the N-ter of engineered protein in comparison with the wild type protein, also suggests that in absence of $\mathrm{C}$-ter peptide, the restriction of movement on $\mathrm{N}$-ter is eliminated. Despite 
the more fluctuations observed in $\mathrm{C}$ - and $\mathrm{N}$-ter of the engineered protein, the average mobility of the catalytic flap has declined. This observation might point to a possible internal interaction between the catalytic flaps in the absence of C-ter peptides.

\section{Carboxyl terminal peptide interactions}

During the MD simulation, various interactions between terminal peptides and catalytic flaps of both 604isoform monomers were observed. Due to the disordered nature of these peptides, the interactions between $\mathrm{C}$-ter peptide of one monomer and $\mathrm{N}$-ter, catalytic flap and carboxyl peptide of the other monomer was not unexpected. As shown in figure 4, theC-ter (blue) of the lower monomer, over the course of MD simulation, was extended and reached to the catalytic flap (magenta) of upper monomer, while still partly covering catalytic flap ofthe same monomer. This interaction was limited to the lower monomer and the extension of $\mathrm{C}$-ter peptide of the upper monomer to reach the catalytic flap of adjacent monomer did not occur. Instead, this peptide covered the catalytic flap of the same monomer, limiting its mobility. This observation is consistent with the low RMSF of the catalytic flaps.

Figure 5 illustrates details of interaction setween C-ter and catalytic flap of distinct 604-isoform monomers. In this interaction hydrogen bonds between Gly472, Ser473 and Asp475 of catalytic flap and Thr577, Cys576 and Ser565 of the C-ter peptide of the other monomer were formed, respectively. This interaction might highlight the possible role of $\mathrm{C}$-terminal peptide in attenuation of the catalytic activity among retinal isoforms.

As mentioned in previous sections, interactions between the $\mathrm{C}$ - and $\mathrm{N}$-ter peptides of the 604 -isoform within the same monomer were observed. These terminal peptides formed two hydrogen bonds between Glu2 of the N-ter peptide and Arg592 of the C-ter peptide and a hydrogen bond between Glu2 and Gly593

(Fig. 6). This explains the lower values of RMSF in N-ter of the 604-isoform. A contact between Glu10 and Arg398 has previously been reported [14]. Along with this finding, interactions between amino terminal peptide and the rest of the protein were not observed.

\section{Interactions in absence of C-terminal peptide}

Catalytic flap, not restricted by the presence of C-ter, showed higher mobility. During the MD simulation, for short periods of time interactions between catalytic flaps of engineered monomers were observed. These interactions resulted in forming a hydrogen bond between Lys493 and Asp461 of the catalytic flaps of the upper and lower monomers (Fig. 7). This interaction lasted for the majority of simulation time, leading to lower RMSF value of the catalytic flap in comparison to the wild type isoform in which the corresponding domains have been restricted by the $\mathrm{C}$-ter peptides.

Eliminating the mobility inhibition, caused by $\mathrm{C}$-ter peptide on $\mathrm{N}$-ter peptide, in the engineered 604isoform, the N-ter peptide extended and formed a short helix in the hinge connecting CBS and catalytic 
domain. This helix which formed in the hinge of both monomers, occupied the GTP2 binding site. Even though there were no hydrogen bonds formed between the newly formed helix of N-ter and other parts of the protein, the N-ter peptide tends to occupy the binding site of GTP2 (Fig. 8). D283N mutation (equivalent to $\mathrm{D} 226 \mathrm{~N}$ in canonical numbering) which is located in this binding site, has been identified to be effective in occurrence of RP symptoms. Given the importance of this binding site, it might be suggested that in absence or engagement of $\mathrm{C}$-ter peptide, the $\mathrm{N}$-termainal extension could play a regulatory role in catalytic activity of the 604 -isoform.

\section{Discussion}

IMPDH1, a regulatory enzyme in balancing guanine and adenine nucleotide pool size, is a house-keeping enzyme, however, the expression of the IMPDH1 canonical isoform in photoreceptors is undetectable [2, 3 ,25]. Studies have revealed the existence of two retinal isoforms of IMPDH1, originated from alternative splicing $[4,5]$. These isoforms have been identified as the primary reason of the retina-specific mutational phenotypes [1]. Although, some studies reported indistinguishable catalytic activity among canonical and retinal isoforms [26], other published data revealing the varied catalytic activity and mode of inhibition suggested a possible undiscovered role of terminal peptides [14, 27-30]. Despite the growing research on the role of IMPDH1 retinal isoforms in RP, our knowledge about significance of each domain in enzyme's overall activity is still limited.

The present study, performing proteolysis on a recombinant 604-isoform lacking c-terminal peptide and simulating molecular dynamics of a modeled dimer of it, has investigated the potential interactions of $\mathrm{N}$ terminal peptide and change of conformation. Our results confirm previous computational and structural studies [14]. Moreover, formation of a novel short helix in N-terminal of the engineered protein has been suggested.

Proteolytic digestion of the investigated proteins with a-Chymotrypsin resulted in time-dependent intensification of bands on SDS-PAGE qualification in which the engineered protein digested bands appeared in faster time than the wild type isoform. This observation indicates the extended conformation of the engineered protein leading to a more surface accessibility of the protease to digestion sites. Since both experiments produced peptides with similar molecular weight, it can be concluded that C-ter peptide covers the digestion site.

In confirmation of previous studies $[11,12,14]$, we have also encountered the interaction between catalytic flaps. This interaction could be taken as the cause of lower RMSF value and hence higher rigidity of them in comparison to wild type dimer. Although, C-ter peptide restricted the mobility of catalytic flaps, resulting in low RMSF values, still, higher freedom in movement is observed than the corresponding residues in engineered protein. It can be concluded that this interaction is favorable and a potent cause of lower rate of catalytic activity.

Previous studies have identified three nucleotide binding sites [12]. While some published results suggest the occupation of third nucleotide binding site with GTP as the critical site, others point out that 
occupation of first and second binding sites with GTP is enough for inhibition of the enzyme's catalytic activity [14]. Also, mutations identified in nucleotide binding sites cause RP symptoms. A recent study, also, has proposed an anti-inhibitory role for the $\mathrm{N}$-ter peptide of Homo sapiens 595 isoform. This behavior has been interpreted to be dependent on filament assembly and suggested that even in presence of GTP molecules, N-ter peptide prevents the full inhibition of the enzymatic activity [15]. Our computational results suggest that in absence of C-ter peptide, Ala23-Glu28 (604-isoform numbering) form a helix in second nucleotide binding site. Since we found no direct interactions between CBS subdomain and $\mathrm{N}$-terminal peptide, this observation might suggest either a GTP-independent inhibition of the enzyme or masking of the inhibition site which results in higher activity due to the GTP binding prohibition. These hypotheses remain to be studied further.

\section{Conclusion}

In conclusion, the proteolytic digestion of both native 604- and the engineered isoforms indicated a higher accessibility of a-Chymotrypsin to digestion sites due to removal of C-ter peptide. Moreover, our computational results revealed a unique helix formation in $\mathrm{N}$-ter extension. Even though, a 604-isoform lacking $\mathrm{C}$-ter is not naturally expressed in photoreceptor cells, in case of elimination of restricting effect of this terminal peptide on $\mathrm{N}$-ter extension, an internal inhibition of activity is assumed. The accuracy of this hypothesis remains to be tested.

\section{Declarations}

\section{Funding}

The financial support of this investigation has been provided by the research council of University of Tehran.

\section{Competing interests}

All authors declare that they do not have financial interests.

\section{Author Contributions}

All authors contributed to the study conception and design. Material preparation, data collection and analysis were performed by Parisa Elyasi Ebli, Sajjad Gharaghani and Razieh Yazdanparast. The first draft of the manuscript was written by Parisa Elyasi Ebli and all authors commented on previous versions of the manuscript. All authors read and approved the final manuscript.

\section{Data Availability}

Data presented in the submitted manuscript are available upon reasonable request.

\section{Ethics approval}


Not relevant

\section{Consent to participate}

Not relevant

\section{Consent to publish}

None

\section{Acknowledgments}

The authors appreciate the financial support of this investigation by the Research Council of the University of Tehran.

Molecular graphics and analyses performed with UCSF Chimera, developed by the Resource for Biocomputing, Visualization, and Informatics at the University of California, San Francisco, with support from NIH P41-GM103311.

\section{References}

1. Bowne, S. J., Sullivan, L. S., Mortimer, S. E., Hedstrom, L., Zhu, J., Spellicy, C. J., Gire, A. I., HughbanksWheaton, D., Birch, D. G., Lewis, R. A., Heckenlively, J. R., \& Daiger, S. P. (2006). Spectrum and frequency of mutations in IMPDH1 associated with autosomal dominant retinitis pigmentosa and leber congenital amaurosis. Investigative ophthalmology \& visual science, 47(1), 34-42. https://doi.org/10.1167/iovs.05-0868

2. Majd, N., Sumita, K., Yoshino, H., Chen, D., Terakawa, J., Daikoku, T., ... Sasaki, A. T. (2014). A review of the potential utility of Mycophenolate Mofetil as a cancer therapeutic. Journal of Cancer Research, 2014, 1-12. doi:10.1155/2014/423401

3. Magasanik B., Moyed H. S., \& GEHRING, L. B. (1957). Enzymes essential for the biosynthesis of nucleic acid guanine; inosine 5'-phosphate dehydrogenase of Aerobacter aerogenes. The Journal of biological chemistry, 226(1), 339-350.

4. Konno, Y., Natsumeda, Y., Nagai, M., Yamaji, Y., Ohno, S., Suzuki, K., \& Weber, G. (1991). Expression of human IMP dehydrogenase types I and II in Escherichia coli and distribution in human normal lymphocytes and leukemic cell lines. The Journal of biological chemistry, 266(1), 506-509

5. Baykov, A. A., Tuominen, H. K., \& Lahti, R. (2011). The CBS domain: a protein module with an emerging prominent role in regulation. ACS chemical biology, 6(11), 1156-1163. https://doi.org/10.1021/cb200231c

6. Hedstrom L. (2012). The dynamic determinants of reaction specificity in the IMPDH/GMPR family of $(\beta / a)(8)$ barrel enzymes. Critical reviews in biochemistry and molecular biology, 47(3), 250-263. https://doi.org/10.3109/10409238.2012.656843 
7. Glanser ME, Gerlt JA, Babbitt PC. (2006) Evolution of enzyme superfamilies. Curr Opin Chem Biol. 2006; 10:492-7.

8. Soskine, M., \& Tawfik, D. S. (2010). Mutational effects and the evolution of new protein functions. Nature reviews. Genetics, 11(8), 572-582. https://doi.org/10.1038/nrg2808

9. Labesse $G$, Alexandre T, Vaupre $L$ et al (2013) MgATP regulates allostery and fiber formation in IMPDHs. Structure 21(6):975-985. https ://doi.org/10.1016/j.str.2013.03.011

10. Min D, Josephine HR, Li H, Lakner C, MacPherson IS, et al. (2008) An Enzymatic Atavist Revealed in Dual Pathways for Water Activation. PLOS Biology 6(8): e206. https://doi.org/10.1371/journal.pbio.0060206

11. Buey, R. M., Ledesma-Amaro, R., Velázquez-Campoy, A., Balsera, M., Chagoyen, M., de Pereda, J. M., \& Revuelta, J. L. (2015). Guanine nucleotide binding to the Bateman domain mediates the allosteric inhibition of eukaryotic IMP dehydrogenases. Nature communications, 6, 8923. https://doi.org/10.1038/ncomms9923

12. Buey, R. M., Fernández-Justel, D., Marcos-Alcalde, Í., Winter, G., Gómez-Puertas, P., de Pereda, J. M., \& Luis Revuelta, J. (2017). A nucleotide-controlled conformational switch modulates the activity of eukaryotic IMP dehydrogenases. Scientific reports, 7(1), 2648. https://doi.org/10.1038/s41598-01702805-x

13. Johnson, M. C., \& Kollman, J. M. (2020). Cryo-EM structures demonstrate human IMPDH2 filament assembly tunes allosteric regulation. eLife, 9, e53243. https://doi.org/10.7554/eLife.53243

14. Andashti, B., Yazdanparast, R., Barzegari, E., \& Galehdari, H. (2020). The functional impact of the $\mathrm{C} / \mathrm{N}$-terminal extensions of the mouse retinal IMPDH1 isoforms: a kinetic evaluation. Molecular and cellular biochemistry, 465(1-2), 155-164. https://doi.org/10.1007/s11010-019-03675-9

15. Burrell, A. L., Nie, C., Said, M., Simonet, J. C., Fernández-Justel, D., Johnson, M. C., Quispe, J., Buey, R. M., Peterson, J. R., \& Kollman, J. M. (2022). IMPDH1 retinal variants control filament architecture to tune allosteric regulation. Nature structural \& molecular biology, 29(1), 47-58. https://doi.org/10.1038/s41594-021-00706-2

16. Nimmesgern E., Fox, T., Fleming, M. A., \& Thomson, J. A. (1996). Conformational changes and stabilization of inosine 5'-monophosphate dehydrogenase associated with ligand binding and inhibition by mycophenolic acid. The Journal of biological chemistry, 271(32), 19421-19427. https://doi.org/10.1074/jbc.271.32.19421

17. Lowry OH, Rosebrough NJ, Farr AL, Randall RJ (1951) Protein measurement with the Folin phenol reagent. J Biol Chem 193(1):265-275

18. Van Der, Spoel D, Lindahl E, Hess B et al (2005) GROMACS: fast, flexible, and free. J Comput Chem 26(16):1701-1718. https://doi.org/10.1002/jcc.20291.

19. Huang, J., \& MacKerell, A. D., Jr (2013). CHARMM36 all-atom additive protein force field: validation based on comparison to NMR data. Journal of computational chemistry, 34(25), 2135-2145. https://doi.org/10.1002/jcc.23354 
20. Jorgensen, W., Chandrasekhar, J., Madura, J., Impey, R., \& Klein, M. (1983). Comparison of simple potential functions for simulating liquid water. The Journal Of Chemical Physics, 79(2), 926-935. doi: $10.1063 / 1.445869$

21. Haug, E., Arora, J., \& Matsui, K. (1976). A steepest-descent method for optimization of mechanical systems. Journal Of Optimization Theory And Applications, 19(3), 401-424. doi: $10.1007 /$ bf00941484

22. Berendsen, H., Postma, J., van Gunsteren, W., DiNola, A., \& Haak, J. (1984). Molecular dynamics with coupling to an external bath. The Journal Of Chemical Physics, 81(8), 3684-3690. doi: $10.1063 / 1.448118$

23. Parrinello, M., \& Rahman, A. (1981). Polymorphic transitions in single crystals: A new molecular dynamics method. Journal Of Applied Physics, 52(12), 7182-7190. doi: 10.1063/1.328693

24. Pettersen EF, Goddard TD, Huang CC, Couch GS, Greenblatt DM, Meng EC, Ferrin TE. (2004) UCSF Chimera-a visualization system for exploratory research and analysis. Journal of computational chemistry, 25(13), 1605-1612. https://doi.org/10.1002/jcc.20084.

25. Gunter JH, Thomas EC, Lengefeld $\mathrm{N}$ et al (2008) Characterization of inosine monophosphate dehydrogenase expression during retinal development: differences between variants and isoforms. Int J Biochem Cell Biol 40(9):1716-1728. https://doi.org/10.1016/j.bioce I.2007.12.018

26. Xu D, Cobb G, Spellicy C et al (2008) Retinal isoforms of inosine 5-monophosphate dehydrogenase type 1 are poor nucleic acid binding proteins. Arch Biochem Biophys 472:100-104. https://doi.org/10.1016/j.abb.2008.02.012

27. Carr SF, Papp E, Wu JC, Natsumeda Y (1993) Characterization of human type I and type II IMP dehydrogenases. J Biol Chem 268:27286-27290.

28. Hager P, Collart F, Huberman E, Mitchell BS (1995) Recombinant human inosine monophosphate dehydrogenase type I and type II proteins. Purification and characterization of inhibitor binding. Biochem Pharmacol 49(9):1323-1329. https://doi.org/10.1016/0006-2952(95)00026 -v.

29. Mortimer SE, Hedstrom L (2005) Autosomal dominant retinitis pigmentosa mutations in inosine $5^{\prime}$ monophosphate dehydrogenase type I disrupt nucleic acid binding. Biochem J 390:41-47. https ://doi.org/10.1042/bj200 42051

30. Wang XT, Mion B, Aherne A (1812) Engel PC (2011) Molecular recruitment as a basis for negative dominant inheritance? Propagation of misfolding in oligomers of IMPDH1, the mutated enzyme in the RP10 form of retinitis pigmentosa. Biochim Biophys Acta 11:1472-1476. https ://doi.org/10.1016/j.bbadi s.2011.07.006

\section{Figures}




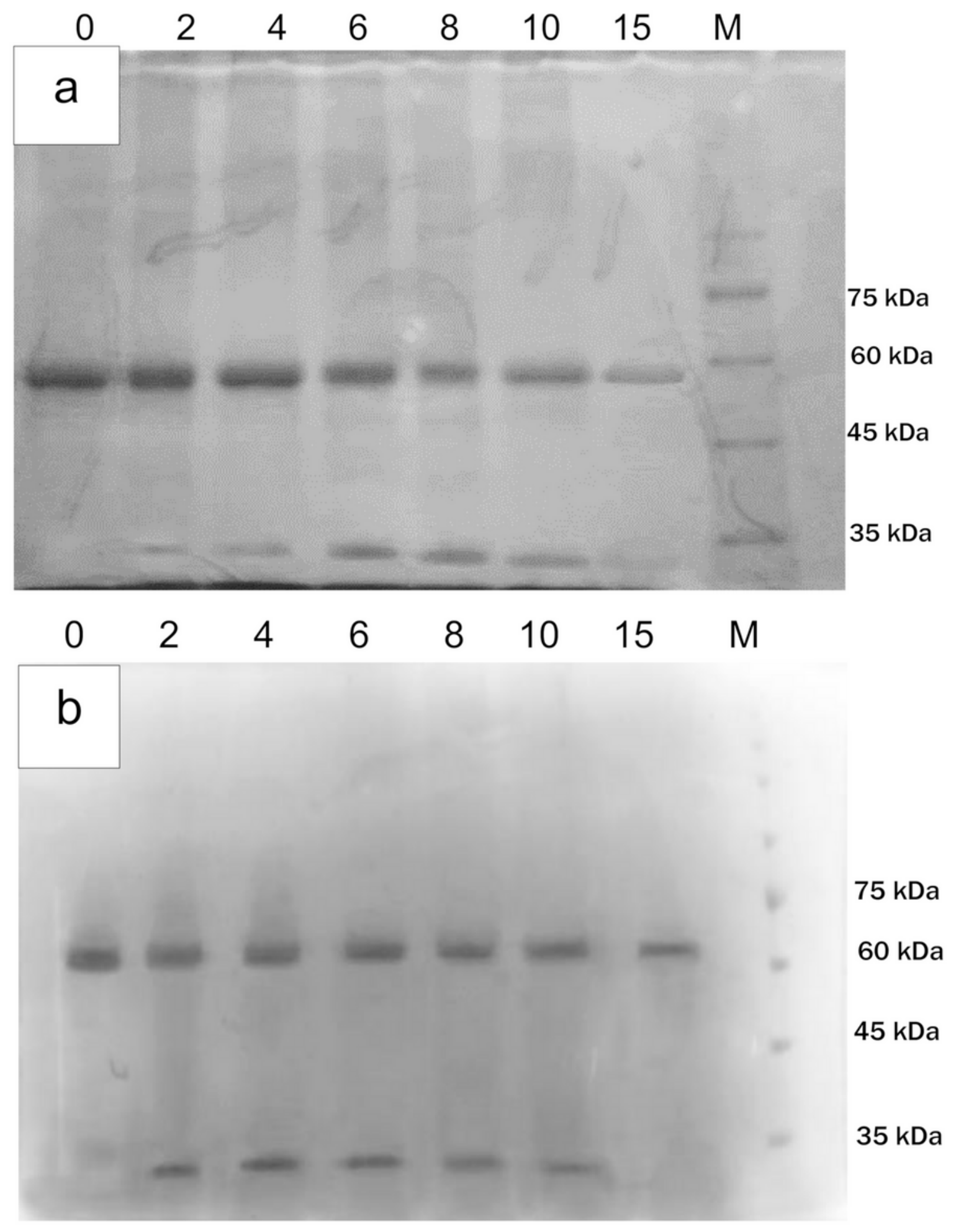

Figure 1

Proteolysis of wild type 604- (a) and engineered (b) proteins. The proteolytic digestion was followed for 0 , $2,4,6,8,10$ and 15 minutes 


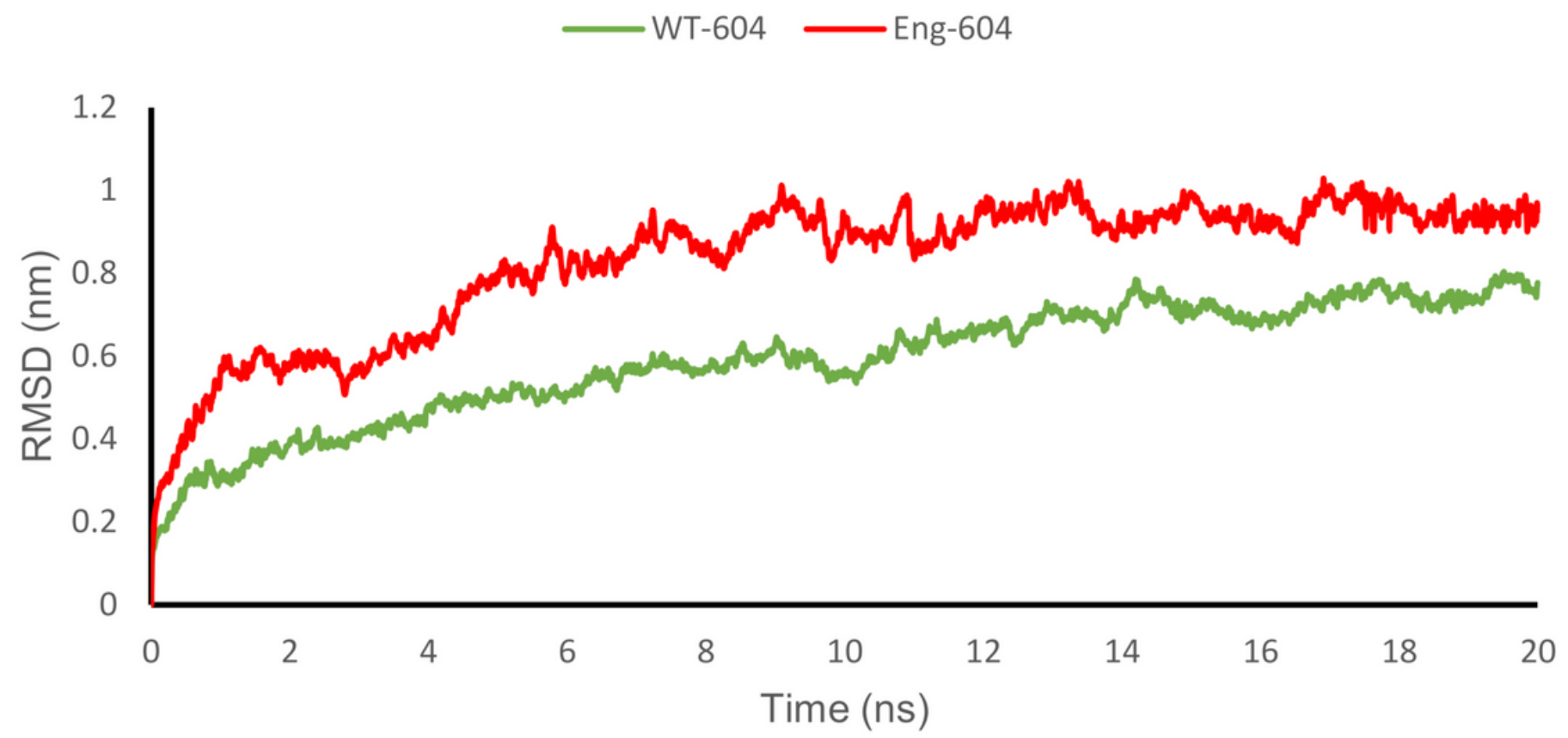

Figure 2

RMSD plot of wild type 604-isoform dimer (WT-604) and engineered (Eng-604) protein models

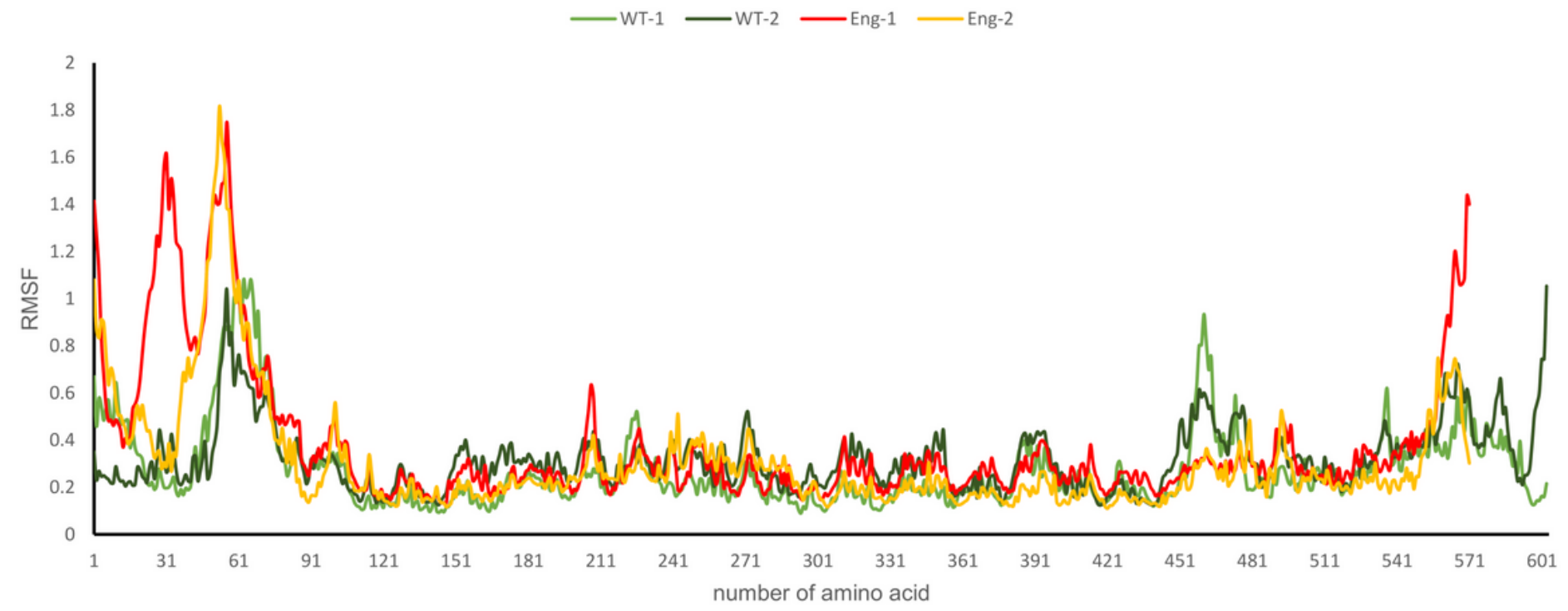

Figure 3

RMSF plot of wild type 604-dimer (WT-1, -2) and engineered protein (Eng-1, -2) 


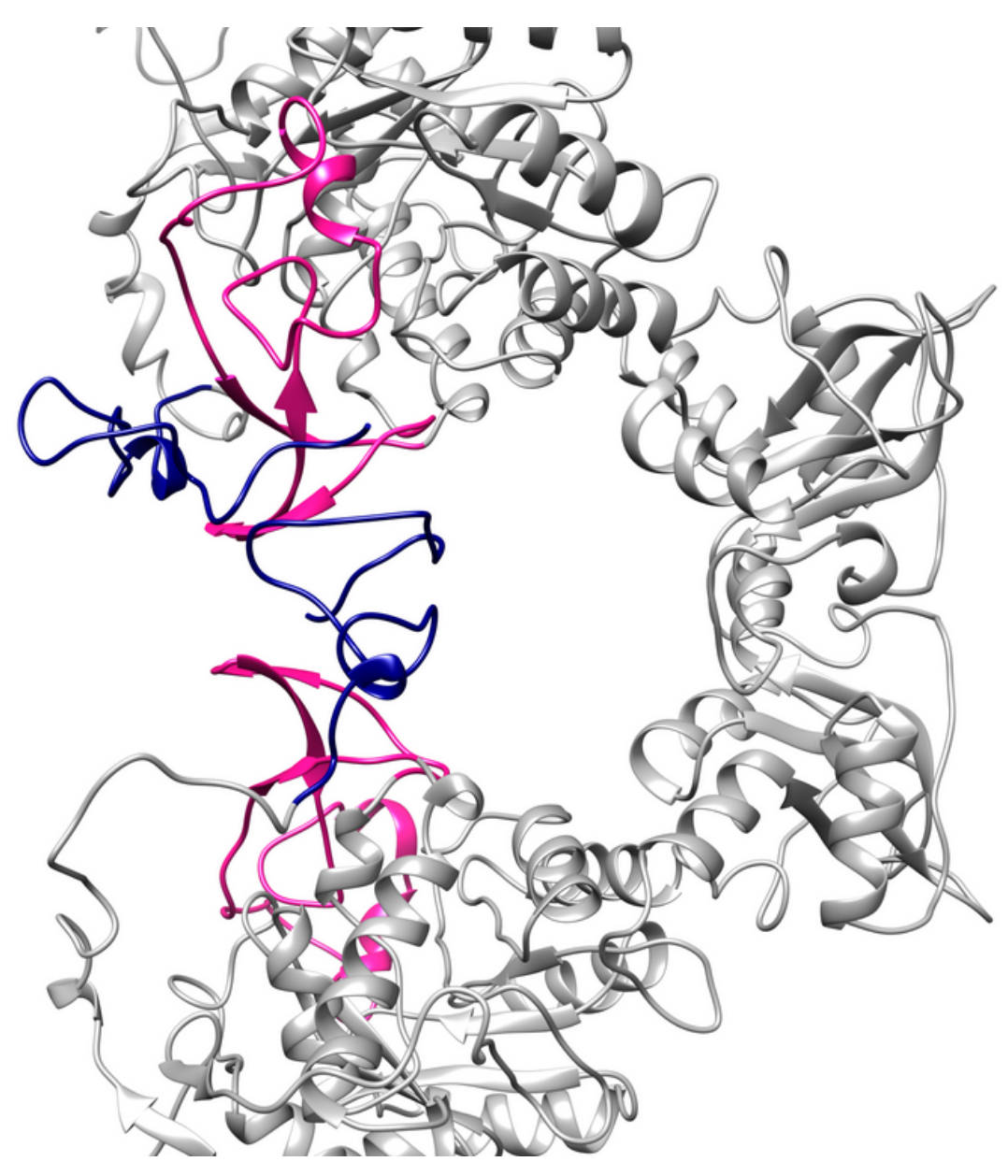

Figure 4

An illustration of interaction between Catalytic flap and C-terminal peptides of upper and lower Wild type 604-isoform. (Magenta: Catalytic flaps, Blue: C-terminal peptide). Here, in order to simplify the visualization of interactions, $\mathrm{N}$-ter peptide has been omitted 


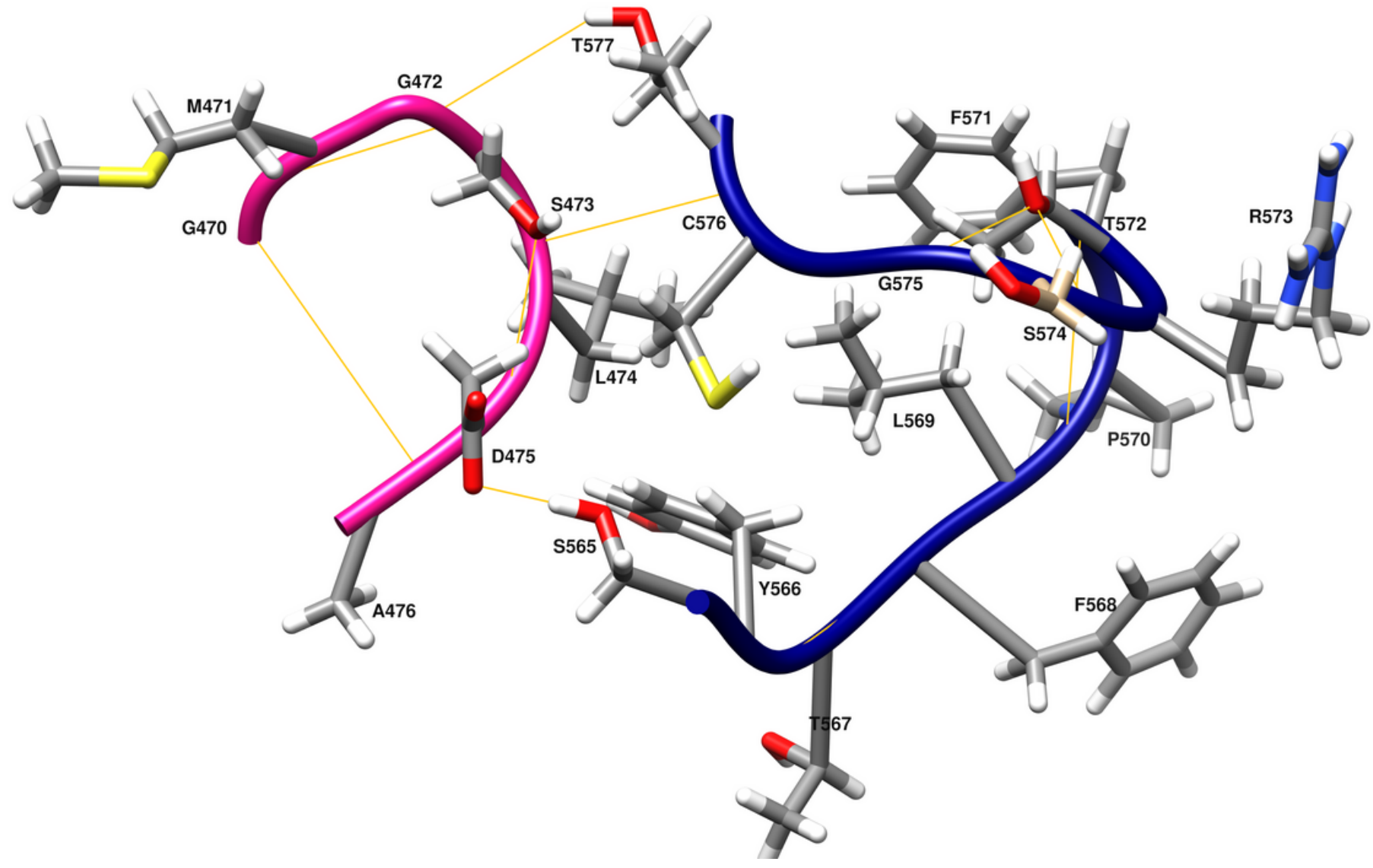

Figure 5

interactions of catalytic flap and C-ter peptide of distinct wild type 604-isoform monomers 


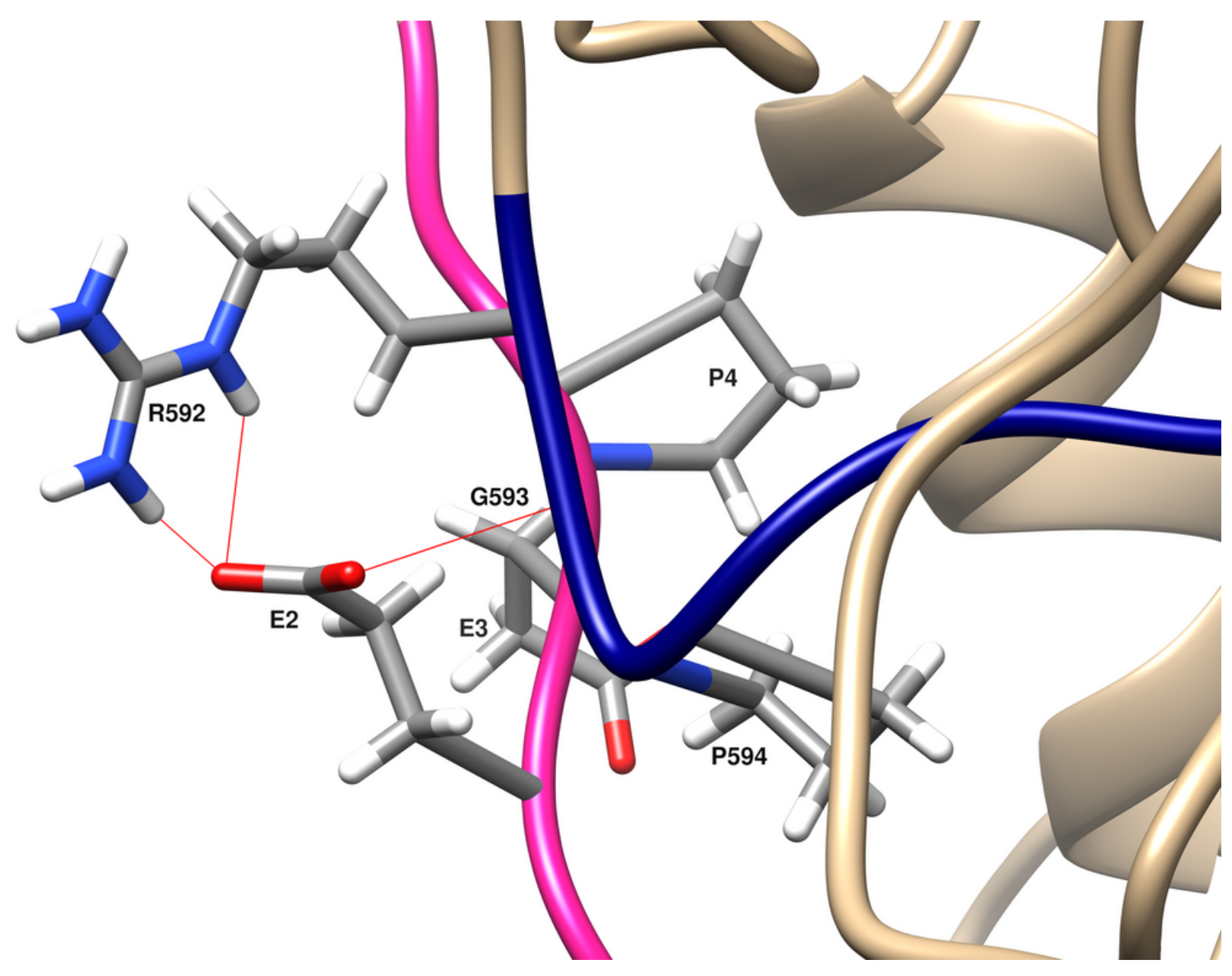

Figure 6

Interactions of $\mathrm{N}$ - and $\mathrm{C}$-terminal peptides within the same monomer 


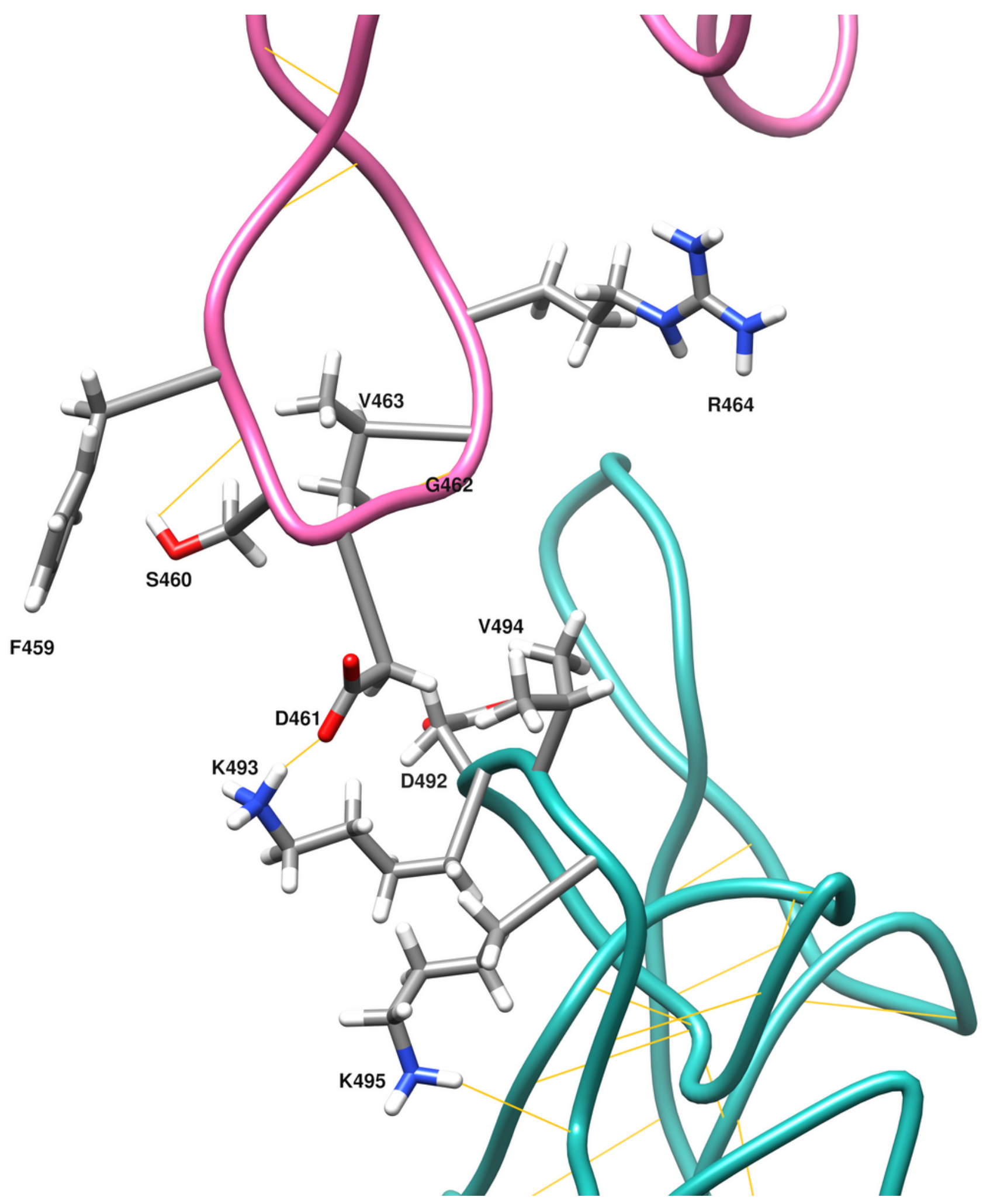

Figure 7

Catalytic flaps of upper and lower monomers of engineered protein interact in the absence of C-ter peptide 


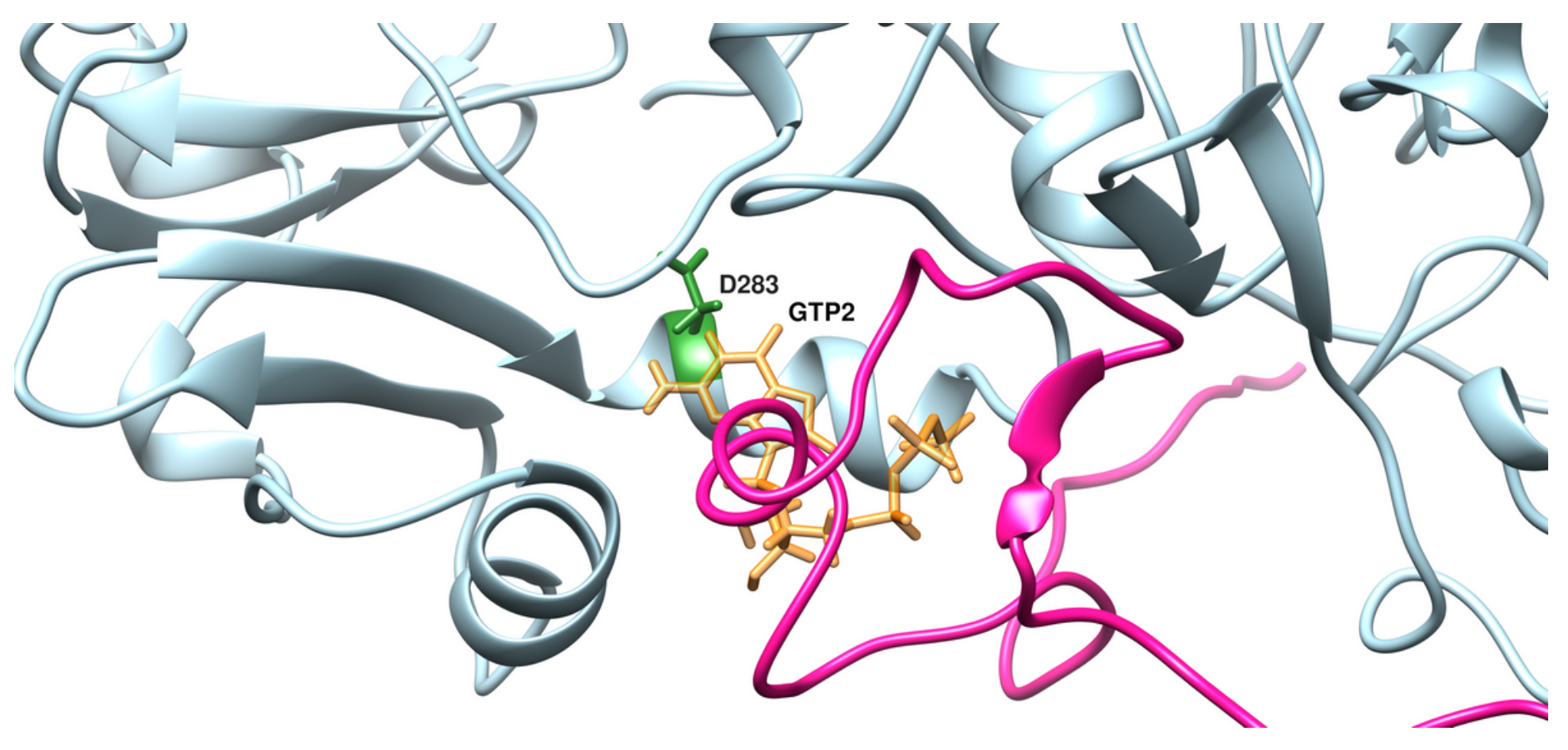

Figure 8

Formation of a short helix in GTP2 binding site. (Magenta: N-ter peptide, Green: Asp283, Orange: GTP) 